\title{
Kluges Konstellationen. Alexander Kluges Fortsetzung der Kritischen Theorie mit narrativen Mitteln
}

Constellations klugiennes: Alexander Kluge ou la continuation de la Théorie critique avec les moyens de la narration

Kluge's constellations. Alexander Kluge's continuation of Critical Theory by other narrative means

\section{Bert-Christoph Streckhardt}

\section{OpenEdition \\ Journals}

Édition électronique

URL : http://journals.openedition.org/ceg/1091

DOI : $10.4000 /$ ceg. 1091

ISSN : 2605-8359

\section{Éditeur}

Presses Universitaires de Provence

Édition imprimée

Date de publication : 18 décembre 2015

Pagination : $55-68$

ISBN : 979-1-03200-020-5

ISSN : 0751-4239

\section{Référence électronique}

Bert-Christoph Streckhardt, « Kluges Konstellationen. Alexander Kluges Fortsetzung der Kritischen Theorie mit narrativen Mitteln », Cahiers d'Études Germaniques [Online], 69 | 2015, Online erschienen am: 17 Dezember 2017, abgerufen am 25 November 2020. URL : http://journals.openedition.org/ceg/ 1091 ; DOI : https://doi.org/10.4000/ceg.1091 


\title{
Kluges Konstellationen. Alexander Kluges Fortsetzung der Kritischen Theorie mit narrativen Mitteln
}

\author{
Bert-Christoph STRECKHARDT
}

Universität Tübingen

„Er ist eine Vertrauensperson für mich, bis heute“, sagt Alexander Kluge über Theodor W. Adorno. Da er sich nicht so ausdrücke wie dieser, klingen seine Texte zwar nicht nach Adorno. Und dennoch macht er die Musik. Auf diese Verbindung in seinem Schaffen, die, wie sich zeigen wird, neben biographischen auch ästhetische und theoretische Bahnen aufweist, legt Kluge deshalb großen Wert: „Es ist mein Text, in dem er anwesend ist, wie er in meinem Leben anwesend ist. [D]ie Gravitation kommt aus seiner Zuverlässigkeit" ${ }^{\text {"1 }}$. Buchstäblich um gravitative Kräfte soll es auch im Folgenden gehen.

Um eines eindringlich vorwegzunehmen: Keineswegs geht es darum, Kluge auf Teufel komm raus in die philosophischen Bibliotheken einzuspeisen. Im Gegenteil geht es, übrigens ganz im klugeschen Sinne, darum, die Philosophie zu entriegeln, akademisch-diskursive Zirkel aufzubrechen und ihr zusätzliche Ausdrucksmittel und Kommunikationswege, sozusagen außerdisziplinäre Medien aufzuzeigen, die es gewiss nicht zu imitieren gilt, mit denen es sich jedoch zu kooperieren lohnt. Will Philosophie, will Kritische Theorie gesellschaftsrelevant sein - und aus diesem, ihrem Selbstverständnis zieht sie schließlich ihre Daseinsberechtigung -, dann darf sie nicht exklusiv sein.

„Philosophie ist nichts Abstraktes“2 , stellt Kluge klar: „Sie geht um mit dem, was für mein Leben relevant ist.“ Damit hat er gewiss Recht. Das Problem aber ist: Sie spricht abstrakt. Dabei tritt sie nicht selten prätentiös bis elitär auf, was eine gehörige Menge an Menschen ausgrenzt - um die aber geht es letztendlich. Im Gespräch am Rande der Kluge-Rétrospective/Prospective in der Cinémathèque française sah er sich mit der Frage konfrontiert, ob er eigentlich so etwas wie ein Brückenbauer sei zwischen Stadt und abgehängter Provinz, jemand, der die Philosophie vom Berg herunter zu den Bürgern bringe. Gleich mit seinem ersten Antwortsatz trat er selbst zurück, um dem Eigentlichen Platz zu machen: „Kann sein, kann aber auch nicht sein. Entscheidend ist: Dort war die Philosophie immer schon." Das stimmt, da kommt sie eigentlich her. Dass man jedoch darauf hinweisen muss, offenbart, wie

1 Peter Laudenbach, „,Wir sind Glückssucher“, im Gespräch mit Alexander Kluge“, in Der Tagesspiegel, 13.02.2012. URL: http://www.kluge-alexander.de/aktuelles/details/artikel/wir-sindglueckssucher.html [Stand: 08.05.2013].

2 Ibid. 
weit die Philosophie sich entfernt hat in ihrer Außenwahrnehmung. Das Staunen des Menschen, das Fragen nach dem Sinn des Lebens ist der Anbeginn von Philosophie. Und von der menschlichen Kulturtechnik „Sprache“: Eine genuine Grundeigenschaft des Menschen bei seiner Suche nach Identität und Sinn stellt das Narrative dar. Der Zugang zum Erfahrungsschatz, den die Welt bereithält, ist narrativ geprägt, gerade die ersten Informationen erfährt man durch Gleichnisse, Märchen und „Stories“, die in der Familie über Generationen hinweg weitergegeben werden. Die Realität ist narrativ vorgeprägt und beeinflusst die eigene Sinneserfahrung. Von der Höhlenmalerei über Mythen und Märchen bis zu den Kommunikationsmitteln der Neuen Medien - seit jeher erzählen sich die Menschen ihre Geschichten, ihre Erlebnisse und Empfindungen.

Der Grund, weshalb diese recht trivialen Bemerkungen Erwähnung finden, ist die folgende Aussage Kluges: Durch das narrative Element nämlich bekomme man „erst eine Differenz in die Sprache“, mit der die „Philosophie überhaupt eine Ausdruckswelt“ gewinne. Man könne „nicht versuchen“, Philosophie ,allein in Hochsprache“ einzufangen. Das ,allein“ heißt also, er rüttelt nicht an der Philosophie, auch nicht an Universität und Wissenschaft, nein er achtet dies in hohem Maße. Stattdessen ist es ihm um Öffnung und Kooperation gelegen:

\begin{abstract}
In der Hinsicht ist also die Begleitung von Philosophie und die Vernetzung von Philosophie mit Erzählung mehr als die Beispiele zur Philosophie (sic!). [...] Es kommt erst Horkheimer und dann kommt Adorno. Jede dieser Erzählungen von denen lässt sich in dieser Weise fortsetzen - zu mehreren. Und dass ich das tue, ist ganz sicher, also das kann ich Ihnen garantieren. ${ }^{3}$
\end{abstract}

Seine Texte und Filme sind also auch nicht bloß irgendwelche Illustrationen von philosophischen Thesen, sondern sie sind autonom. Dies bedeutet für die KlugeRezeption ein großes Glück: Vielpoligkeit. Es ist ein Leichtes, ihn mit anderen Künstlern in Verbindung zu bringen, ja er selbst weist ausdrücklich auf all jene „Gewährsmänner“ und „Vertrauenspersonen“ hin. Gleichzeitig müssen sich die Geisteswissenschaften vor dem „unwissenschaftlichen“ Vorwurf der Beliebigkeit schützen. Gibt es also etwas Grundlegendes, sozusagen Deduktives, das Kluges Arbeiten auch theoretisch verbindet, an dem man sich zur Not festhalten kann, wenn man sich von der intertextuellen Schwerelosigkeit im Kluge-Kosmos treiben lässt und plötzlich von der Schwerkraft eines Seminarraums eingefangen wird?

Die Antwort lautet: Ja. Alexander Kluges Werk lässt sich - trotz oder gerade wegen aller Vielstimmigkeit - als Zusammenhang, gewissermaßen als Physiognomie eines philosophischen Denkzusammenhangs rekonstruieren. Und der ist mit der Chiffre „Kritische Theorie“ am kompaktesten zusammengefasst. Diese unternimmt bekanntlich eine Analyse und Kritik von Gesellschaft, insbesondere hinsichtlich ihrer Macht-, Gewalt- und Freiheitsverhältnisse unter dem handlungsorientierten Leitstern, „dass Auschwitz nicht noch einmal sei“"4. Die Kritische Theorie

3 So Kluge in einem persönlichen Thekengespräch am 27. April $2013 \mathrm{im} \mathrm{,51“} \mathrm{der} \mathrm{Cinémathèque}$ française, Paris.

4 Theodor W. Adorno, „Erziehung nach Auschwitz“, in ders., Erziehung zur Mündigkeit. Vorträge und Gespräche mit Hellmut Becker 1959-1969, hrsg. v. Gerd Kadelbach, Frankfurt am Main, Suhrkamp, 1971, S. 88. 
denkt Kant, Hegel, Marx, Freud und andere zusammen, ist ihren wesentlichen programmatischen Äußerungen nach undogmatisch und selbstreflexiv, ist antikapitalistisch, ideologiekritisch und antiautoritär. Entscheidend ist dabei, dass die Kritische Theorie kein geschlossenes System ist, sondern in erster Linie eine Lebenshaltung. Auf Kluge bezogen darf dabei ein methodisches Problem nicht unerwähnt bleiben: Sein theoretisches und sein künstlerisches Werk stehen nicht einfach im Verhältnis von Theorie und Anwendung. Ziel ist also nicht der Versuch ästhetischer Darstellung von Theorie. ${ }^{5}$ Vielmehr partizipieren auch die theoretischen Schriften Kluges an seiner literarischen Praxis und umgekehrt. Ja eigentlich kann man nicht einmal solche Einteilungen vornehmen, da die dicken Bände mit Oskar Negt noch voller Poesie stecken und selbst der entlegendste Minutenfilm Theoretisches transportiert. Vielleicht liegt genau hier der besondere Reiz von Kluge: analytisch und ästhetisch zugleich am Werk zu sein. Es scheint, dass Form und Inhalt, theoretische Reflexion, intellektuelle Intervention und nie rein-ästhetische Produktion (vielmehr: praktisches Arbeiten) im Gesamtprozess wahrgenommen werden müssen, will man etwas „Wahres“ aussagen. Um es mit seinem eigenen Vokabular zu verdeutlichen: Die Lücken und Bruchstellen im Werk sind „durchlässige“ und „reiche [...] Nahtstellen“, weil sie eine komplementäre, wechselseitige Durchdringung von „spontan (idiosynkratisch)“ und „bewusst (kritisch)“ ermöglichen. ${ }^{6}$

Wenn man Kluge deshalb so etwas wie „Systematik“ andichten wollte, bestünde eine ähnliche Gefahr als wenn man ihm vorschnell „Unsystematik“ vorwürfe. Sein „System“ ist ein nach allen Seiten offenes und dennoch in sich stimmiges AntiSystem und deshalb treffender als „Zusammenhang“, als Kunst des Zusammenhangs zu bezeichnen. Jene Kunst wiederum besteht in einer Kompositionsleistung subjektiv-objektiver Gravitationen.

Gerda raffte sich hoch, [...] schob ihre Brut die sechs Steintreppenstufen zur Geräteecke hinunter, dasjenige in diesem Häuschen, was noch am ehesten etwas Kellerartiges hatte. Es lag anderthalb Meter unter Straßenhöhe. Sie fühlte sich in ihrem „unausgestatteten Häuschen“ als „Leichtbewaffnete“. Sie glaubte nicht, daß die Gefahr bestand, verschüttet zu werden. Sie hatte, wenn die Sprengkörper fielen, jeweils den Atem lange angehalten, weil sie gehört hatte, daß der Luftdruck der Sprengbomben die Lungenbläschen zerriß, also einen Staudruck in der Lunge herstellen, bis es vorüber war. Sie flüsterte jetzt mit dem Kleinen: Nicht atmen, bitte nicht atmen. Das Flüstern machte den Kleinen nervös. Die Älteste machte dicke Backen, atmete gleichwohl. ${ }^{7}$

Das im Werk Kluges womöglich bedeutsamste philosophische Problem, dem er sich als Erzähler widmet, ist das Subjektiv-Objektive: „Das sind einfach die

5 Dies wäre wohl auch nicht mehr als eine Verwechslung zwischen „Theorieschein“ und Kunstwerk. Vgl. Rüdiger Bubner, Ästhetische Erfahrung, Frankfurt am Main, Suhrkamp, 1989, S. 94f.

6 Die Begriffsgegenüberstellung ist $U M, M P$, S. 993 entlehnt, wo das Subjektiv-Objektive behandelt wird. Die auch im Folgenden verwendete Sigle steht für: Oskar Negt/Alexander Kluge, Der unterschätzte Mensch. Gemeinsame Philosophie in zwei Bänden, Frankfurt am Main, Zweitausendeins, 2001. („MP“ darin für: Maßverhältnisse des Politischen).

7 Auszug aus: Der Luftangriff auf Halberstadt am 8. April 1945, in Alexander Kluge, Neue Geschichten. Hefte 1-18. ,Unheimlichkeit der Zeit‘, Frankfurt am Main, Suhrkamp, 1977, Heft 2, S. 24. 
wirklichen Verhältnisse, subjektiv, objektiv, unverstellt gegeneinander gesetzt. Das nennt man Konstellation. Und das ist meine literarische Methode“"8.

Die Philosophie erklärt in Begriffen, die Oper in musikalischen, die Literatur in sprachlichen, der Film in visuellen, in bewegten Bildern. Die Grenzen der Sprache vor Augen, erscheint das von Kluge praktizierte konstellativ-narrative Verfahren, das gekennzeichnet ist durch Multi- und Intermedialität, infinite Bewegung und Metamorphose, Multiperspektivität und Methodenpluralismus, als geeigneteres Ausdrucksmittel - sowohl um das signifié getreuer abbilden, ja um „das Wirkliche von der bloßen Erscheinung unterscheiden" ${ }^{\text {" } 9}$ zu können als auch in Kommunikation mit anderen darüber treten zu können. Immerhin erreichen Kluges Formen von Kritischer Theorie noch einmal deutlich mehr Menschen als etwa die Bemühungen Adornos und Benjamins im Rundfunk.

Worte Adornos in der Negativen Dialektik beschreiben dieses Erkenntnisund Darstellungsdilemma von Wissenschaft im Allgemeinen und Philosophie im Besonderen:

Konstellationen allein repräsentieren, von außen, was der Begriff im Innern weggeschnitten hat, das Mehr, das er sein will so sehr, wie er es nicht sein kann. Indem die Begriffe um die zu erkennende Sache sich versammeln, bestimmen sie potentiell deren Inneres, erreichen denkend, was Denken notwendig aus sich ausmerzte. ${ }^{10}$

Adorno bietet hier einen konkreten Lösungsvorschlag an: eine konstellative, anti-hierarchische Darstellungsweise. Kluge hat sich dieser Idee angenommen und verwirklicht sie mit den Mitteln der literarischen Moderne und des selbst mitbegründeten Neuen Deutschen Films, etwa durch Montage und Cross-Mapping. Mit Nachdruck: Kluge als „Montagekünstler“ zu bezeichnen, ist zwar korrekt, doch greift es zu kurz - die Montage ist ein, neben der Kurzgeschichte vermutlich das klugesche Werkzeug zur Herstellung von Konstellationen. Sie ,ist vielmehr Ausdruck der Autonomie dessen, was ich beschreibe, der Versuch, etwas schwer Verständliches in seinem Eigenleben zu erhalten; die Liebe zu diesem Eigenleben ist das kommunikative Vehikel. [...] Der Zusammenhang eines Gartens, das ist Montage“. ${ }^{11}$ Die Gärtner-Metapher weist auf das gerade nicht Destruktive der Montage hin, sondern auf ihre affizierend-konstruktive Kraft: „Man betreibt in meinem Sinn also nicht Montage aus der Lust der Zerstückelung, sondern aus Achtung davor, dass etwas von sich selbst wächst" ${ }^{12}$. Ein Hinweis auch auf die mimetische Qualität der Montage.

8 Alexander Kluge, in Claus Philipp, „Ein Kapitän soll aufpassen, wohin er fährt“, in Falter, Wien 6/2012, 08.02.2012, S. 22.

9 Ibid., Bd. 1, S. 18 (Vorwort).

${ }^{10}$ Theodor W. Adorno, Gesammelte Schriften, hrsg. v. Rolf Tiedemann u. Mitwirk. v. Gretel Adorno, Susan Buck-Morss u. Klaus Schultz, Frankfurt am Main, Suhrkamp, 1970ff., Bd. 6, S. 164f. Im Folgenden: AGS. Die Ästhetik begreift Adorno als ,,philosophisch in sich“. „Unverhüllt ist das Wahre der diskursiven Erkenntnis, aber dafür hat sie es nicht; die Erkenntnis, welche Kunst ist, hat es, aber als ein ihr Inkommensurables.“ AGS 7, S. 191.

11 Alexander Kluge, „Der Autor als Dompteur oder Gärtner“, Rede zum Heinrich-Böll-Preis 1993, in ders., Personen und Reden, Berlin, Wagenbach, 2012, S. 26.

12 Ibid. 
Die Idee der Konstellation erweitert Kluge zu einer eigenen, grundlegenden ästhetischen Darstellungsmethode. Weit über das Künstlerische hinaus, erweist sie sich von großer erkenntniskritischer Bedeutung: „Die einzelnen Phänomene, die sich beobachten und beschreiben lassen, sollen gerade ihr Eigenleben behalten. Sie sind primär und von sich aus nicht Instrument eines übergeordneten Sinnzusammenhangs" ${ }^{13}$. In ihren wechselseitigen Beziehungen und in ihrer Eigenbewegung nehmen sie Gestalt an und werden begreifbar: „Oft stehen sie in Nachbarschaft, Konstellation oder Gegensatz, in Abstoßung oder Anziehung zu etwas anderem“, erklärt Kluge ${ }^{14}$. Doch es gebe „auch Spannungsverhältnisse zwischen Einzelheiten, die gleichgültig gegenüber einem gemeinsamen Sinn bleiben, bloß Parallelen bilden“.

Ein Begriff muss so lange durchgearbeitet werden, theoretisch und rational wie ästhetisch und phantasievoll, bis er beginnt, sich an seinen Rändern dermaßen zu weiten, dass er in den nächsten Begriff übergeht - und so also auf das hinweist, was außer ihm noch existiert. Das bedeutet also, dass die klugesche Konstellationsmethode tatsächlich ein Erkenntnismittel darstellt, weil in ihr erstens der Begriff bzw. ein Problem umfangreich (auch spielerisch) verifiziert wird, zweitens das Identische sowohl auf ein konkretes, ausgeschlossenes Nichtidentisches hinweist, wie drittens überhaupt auf das Mehr an Welt. „Ich halte so lange an dem Wahrheitsanspruch eines Begriffs fest, bis er von sich aus sagt: ,Ich bin nicht die ganze Wahrheit. Ich muss einen weiteren Begriff, einen weiteren Erfahrungszusammenhang aufnehmen“". ${ }^{15}$ Die Konstellation ist deshalb nie nur ein Epistemologe, sondern immer auch ein Kommunikator, ein Vernetzer: Sie vermittelt zwischen den Begriffen wie den Subjekten, zwischen Identischem und Nichtidentischem, zwischen Bekanntem und Fremdem. An diesem Punkt wird somit auch eine soziale, kulturelle und gesellschaftliche Dimension von kritisch-theoretischen Überlegungen ersichtlich.

Folgt man diesen Gedankengängen weiter, so ist der Habitus eines Denkens, das allein (d.h. ohne Reflexion) in bestimmten Kategorien einen Gegenstand identifizieren will, zum Scheitern verurteilt. Es geht darum, eine „Sache selbst“ zu begreifen und nicht bloß analytisch-reduktionistisch in irgendein „Bezugssystem“ „ein[zu]passen“ - so die Vorgabe Adornos, der Kluge folgt. ${ }^{16}$ Dabei bedient er sich der akademischen Begriffe ebenso wie der Poesie, doch versucht er nicht, Gedanken und Dinge begrifflich festzuzurren, sondern er umkreist sie assoziativ mit Gleichnissen und Gegensätzen über ferne Zeiten hinweg, ohne dabei etwa auf die Qualitäten von Begriffsgeschichte zu verzichten. Er wendet keine Gewalt an, sondern die, wenn, dann sanfte Gewalt der Hebammenkunst, die des empathiefähigen Feingriffs, der sich auf die Eigenbewegung des Anderen einlässt.

Auch in Geschichte und Eigensinn wird auf dieses Problem hingewiesen: „Arbeitsmittel der Theorie sind analytische Perspektiven und Elementarisierungen, die das, was sich in Wirklichkeit in Bewegung befindet, zergliedern und damit - man

\footnotetext{
13 Ibid., S. 25.

14 Ibid.

15 Oskar Negt, in Alexander Kluge, Was heißt NICHTS?, Kairos Film, 2009, 14 min.

${ }_{16}$ AGS 6, S. 36.
} 
mag so oft man will darauf hinweisen, daß es sich bewegt - die Prozesse anhalten“". ${ }^{17}$ Man beachte den gedanklichen Einschub: Die ausgefeilteste Benennung genügt nicht. Mit einer Analyse ohne Mimesis der sich wandelnden Form tötet man das, was man verstehen will. Alexander Kluge und seine Werkstatt der Autoren nun begleiten deshalb die Philosophie mit der konstellativen Darstellungskraft der literarischen wie filmischen Erzählung, mit dynamischen, infiniten Text- und Bildversatzstücken und der Methode des epischen Bruchs gegen die lineare Dramaturgie, stellvertretend für den selbstbewussten Kampf gegen die scheinbare Fatalität des Schicksals, das es für Kluge gar nicht gibt.

Dies führt uns auf einen weiteren Schlüsselbegriff Kluges, die sogenannte „Bifurkation“. Diese steht erstens, und da korrespondiert sie mit dem kairósGedanken, für die an einer zeitlichen Gabelung Licht gewordene reale Möglichkeit eines anderen Verlaufs der Dinge, die Suche nach Auswegen, ein Aufblitzen einer glücklicheren Welt. Wie viele Menschen wären am Leben geblieben, wenn der 20. Juli 1944, das Attentat auf Hitler, gelaufen wäre wie geplant? Wäre Halberstadt im April 1945 bombardiert worden?

Zweitens ist dieses Bild der bifurcation von ideengeschichtlicher Prägung, also dem Bewusstsein von der mitunter unterirdisch und zeitlich versetzten Wanderung eines in der Geschichte schon einmal so oder so ähnlich Gedachten. Allen voran: Die Idee der Emanzipation des Menschen, die sich historisch betrachtet, trotz ständiger Rückschläge ja sukzessiv weiterentwickelt.

Der seinem Werk inhärente Klang der Zuversicht übertönt deshalb jedes Anstimmen sentimentaler Trauergesänge auf gescheiterte Revolutionen mit unzähligen bestärkenden Beispielen eines Beinahe-Gelingens. Durch das Werk Kluges erhält man erst einmal ein Bewusstsein davon, wie viel Utopievermögen in der Geschichte enthalten ist. Durch das Montieren mehrerer Bilder oder Geschichten in einem Erzählraum werden die Zwischenräume ephemer zugänglich, sodass auch all „das Nichterzählte“ plötzlich ,zu ahnen ist". ${ }^{18}$ In dieser unerzählten Wirklichkeit ist zugleich auch enthalten ein unverwirklichtes Realitätsreservoir an Erfahrungen und Energien. - Und deshalb dürfte der Bifurkationsbegriff die Toleranzgrenze eines streng faktisch arbeitenden Historikers zwar bis aufs Äußerste strapazieren, pauschal als kontrafaktisch bezeichnet werden kann er jedoch auch nicht, da er auf prinzipielle Defizite im Informationsgehalt von Geschichtsschreibung aufmerksam macht.

Kluge spricht von einer Grammatik der historisch gewordenen Erfahrung und Erinnerung, die in den kulturellen Schätzen einer Gesellschaft aufbewahrt sind und also einen langen Atem beweisen, d.h. von einer gewissen Zuverlässigkeit zeugen. Eine solche „Grammatik, die sozusagen im Erzählstrom einer Gesellschaft historisch enthalten ist, ist der äußerlich-sprachlichen Grammatik überlegen“"19,

17 Oskar Negt/Alexander Kluge, Geschichte und Eigensinn. Geschichtliche Organisation des Arbeitsvermögens. Deutschland als Produktionsöffentlichkeit. Gewalt des Zusammenhangs, Frankfurt a. M., Zweitausendeins, 1981, S. 224. Hervorhebung gemäß Original. Im Folgenden: GE.

${ }_{18}$ Vgl. Alexander Kluge, Theorie der Erzählung. Frankfurter Poetikvorlesungen, Berlin, Filmedition Suhrkamp, 2013. „Die Wirklichkeitsmassen, die auf ihre Erzählung warten“ (3. Vorlesung, 19.06.2012).

$19 U M$, Bd. 1, S. 11. 
also der Alltagssprache, der Sprache der Nachrichtensendungen und dem hitzigen Vokabular aller kurzlebigen Schlagzeilen allemal.

Die eigene Erfahrung der Bombe hat sich eingeschrieben in die Arbeit des Halberstädters. Und so schreibt und filmt und redet er wie ein literarischer Abfangjäger gegen alle an, die da noch kommen wollen. Übersicht über hundert Kriege, Übersicht über hundert Auswege: „Es gibt Glücksfälle, die in der Vergangenheit verborgen sind und nicht genutzt wurden, das ist das future antérieure (sic!) “20. Die Zukunft also liegt in der Vergangenheit. Hierfür baue er unentwegt Metaphern, um „die wirklichen Verhältnisse in der Beschreibung so zu verlangsamen, dass die Emotion Kontakt zur Sache aufnehmen kann“. Das Mittel der Metapher interessiert nicht etwa als Redeschmuck, sondern wird hier als Entschleuniger zur Ermöglichung von Erfahrungen gesellschaftsrelevant:

Heiner Müller sagte, Metaphern sind eine Vorkehrung, ein Raster, ein Werkzeug, um eine Erfahrung, die zu schnell auf uns einstürzt, sodass wir sie nicht wahrnehmen können, wenigstens nicht mit allen unseren verschiedenen sinnlichen Fähigkeiten wahrnehmen können, so zu verlangsamen, dass wir sie wahrnehmen. ${ }^{21}$

Durch Verlangsamung des Schrecklichen und Tragischen ist das Unglück, selbst wenn es bereits geschehen ist, doch noch psychologisch einzuholen, ist es $\mathrm{zu}$ verarbeiten. ${ }^{22}$ Insofern hat der Bildersturm der einschlagenden Flugzeuge und einstürzenden Türme weder $\mathrm{zu}$ einem Verstehen noch zu einem Verarbeiten beigetragen. ${ }^{23}$ Es ist nicht einfach, dieser Übermacht der Bildermassen der Gegenwart die „Verankerung in Vorzeiten“ 24 entgegenzusetzen, die scheinbar wenig Anschaulichkeit bereithält.

„Ohne Zeit und rasante Zeitbeschleunigung $\mathrm{zu}$ unterbrechen, ist lebendige Erfahrung von Gegenwart nicht möglich“. ${ }^{25}$ Und so setzt er der Information die Narration entgegen; „Nachrichten von ruhigen Momenten“ als Prinzip. Dass Kluges medienkritische Position der Entschleunigung zugleich immer auch eine erkenntniskritische ist, liegt auf der Hand. Die Kunst der Unterbrechung ist aufgrund ihrer Entschleunigung, ihrer Entdramatisierung ein analytischer Akt.

Auf den Pessimismus Adornos und Horkheimers und den anschließenden Optimismus Habermas' folgt mit Alexander Kluge ein zuversichtlicher Realismus. ${ }^{26}$

\footnotetext{
${ }^{20}$ Alexander Kluge, in Iris Radisch/Ulrich Greiner, ,Der Friedensstifter. Im Gespräch mit Alexander Kluge“, in Die Zeit, 23.10.2003.

${ }^{21}$ Alexander Kluge, in „,,Man kann nicht lernen, nicht zu lernen“ - Alexander Kluge im Unterricht“, in Der Deutschunterricht. Beiträge zu seiner Praxis und wissenschaftlichen Grundlegung, Jg. LXIV, Heft 3/2012, S. 14.

22 Vgl. $U M, M P$, S. 962.

${ }^{23}$ Stattdessen Rückfall in Mythos (,War on Terror“, „Achse des Bösen“) und Zeichen der Angst in Form von Aggression (erstarkter Patriotismus bis zum Nationalismus, totale Überwachung).

${ }^{24}$ Alexander Kluge, 30. April 1945. Der Tag, an dem Hitler sich erschoss und die Westbindung der Deutschen begann, Berlin, Suhrkamp, 2014, S. 288.

${ }^{25} U M, M P, \mathrm{~S} .753$.

${ }^{26}$ D.h. zuversichtlich aufgrund von konkreten Erfahrungswerten: Sicher sei er kein Pessimist, sagt Kluge über sich selbst, aber im Grunde sei er auch kein Optimist. Er sei schlicht Sammler und somit Kenner von unzähligen Auswegen. Mit dieser Nuance eines realistisch-zuversichtlichen Dritten beschützt Kluge seine Arbeiten hier vor Angriffen.
} 
Er weiß von der Inkubationszeit des Emanzipativen. Mit der Haltung eines Nichtsdestotrotz lebt Kluge eine konkret-optimistische Erinnerungskultur vor, die auf das emanzipative Potenzial menschlicher Möglichkeiten vertraut und sie mit historischem Erfahrungsgehalt versorgen will, um sie in ihrem krankheitsanfälligen Entwicklungsprozess davor zu bewahren, in Mythos zurückzufallen:

\title{
Wenn Sie die Irrtümer aller Vergangenheiten hinzufügen, haben Sie etwas mehr Orientierung. Man kann also gar nicht mehr sagen, wir horten Wahrheiten. Sondern wir unterscheiden, was die größten Irrtümer waren, und isolieren das, was wir auf gar keinen Fall mehr wiederholen wollen. ${ }^{27}$
}

Das Ziel muss lauten, allerhand geistige Zugänge zu den Museen und Archiven zu legen, sodass ihre Erfahrungsschätze Einzug in gegenwärtige - insbesondere öffentliche - Reflexionsprozesse halten.

\begin{abstract}
Der Konstellation gewahr werden, in der die Sache steht, heißt soviel wie diejenige entziffern, die es als Gewordenes in sich trägt. Der Chorismos von draußen und drinnen ist seinerseits historisch bedingt. Nur ein Wissen vermag Geschichte im Gegenstand zu entbinden, das auch den geschichtlichen Stellenwert des Gegenstandes in seinem Verhältnis zu anderen gegenwärtig hat; Aktualisierung und Konzentration eines bereits Gewußten, das es verwandelt. Erkenntnis des Gegenstands in seiner Konstellation ist die des Prozesses, den er in sich aufspeichert. ${ }^{28}$
\end{abstract}

Die Idee der Gegenwart dehnt Kluge aus zu der der Gegenwärtigkeit, was einem Sog der Zeiten gleich kommt. Benjamins „Vor- und Nachgeschichte“29 plus die Musilsche und Klugesche Zeitdimension des Optativs - Vergangenes, Zukünftiges, Mögliches und Wünschbares - erweitern die Jetztzeit, wodurch das fatalistische Geschichtskontinuum, jener Überbau objektiver Geschichtsschreibung, regelrecht aufgesprengt, schließlich Energie freigesetzt wird. ${ }^{30}$ Die Erziehung der Eltern etwa oder die Hoffnung auf etwas Zukünftiges beeinflussen schließlich die gegenwärtige Handlung. Kluge regt ein Zeitbewusstsein an, das das Punktuelle von Gegenwart verdickt, den Moment lang hält. Der Zeitpunkt wird zu einem extensionalen Zeitfeld, in dem die Zeiten ungetrennt und gemeinsam wirken. Man erhält eine Entideologisierung der Gegenwart, einen Zeitgewinn, der einen Reflexionsraum schafft mit einer Distanzierung zur Hektik des Jetzt, sodass man sich der geschichtlichen Bedeutung bewusst werden kann, Gegebenheiten einzuordnen weiß. Dies ist also nicht nur von ästhetischer Bedeutsamkeit (durch das Narrativ gelingt die Überführung des Flüchtigen ins Dauerhafte): Durch Montage und Subjektivierung wird auch das Objektive in unmittelbare Reichweite zum Subjektiven, das Entscheidungsferne an den eigenen Zuständigkeitsbereich gesetzt, die Entscheidungsgewalt und mit ihr der Bereich des Möglichen in die Breite gezogen.

${ }^{27}$ Alexander Kluge, in Florian Illies, „Herr Kluge, wie wird das Jahr 2013?“, in Die Welt, 12.01.20013.

28 AGS 6, S. 165.

${ }^{29}$ Walter Benjamin, Gesammelte Schriften, hrsg. v. Rolf Tiedemann u. Hermann Schweppenhäuser, u. Mitwirk. v. Theodor W. Adorno u. Gershom Scholem, Bde. I-VII, Suppl. I-III (17 Bde.), Frankfurt a. M., Suhrkamp, 1972ff., Bd. I, S. 227. Im Folgenden: BGS.

${ }^{30}$ Vgl. Kluge, Theorie der Erzählung, „Die Wirklichkeitsmassen, die auf ihre Erzählung warten“ (3. Vorlesung, 19.06.2012). 
Dergestalt ist eine solche Denkart sowohl ein Abgesang auf Fortschrittsideologie und Kapitalakkumulation als auch Gegenprojekt zu moralisierendem, pseudobetroffenen Denkmaltum. Die Versöhnung von Mythos und Natur, folgt man der Dialektik der Aufklärung, gelingt schließlich durch Erinnerungsarbeit. ${ }^{31}$ Letztlich dient also Arbeit an und mit Erinnerung dem übergeordneten, eigentlichen Ziel: der Befreiung des Menschen aus seiner Unmündigkeit. Schließlich gilt als „eine der edelsten Aufgaben des Denkens“ die „Wiedereinsetzung der Erinnerung in ihr Recht als Mittel der Befreiung“، ${ }^{32}$

Kluge betont oft, wie gefährlich das Subjektive anwachsen kann, wenn es das Objektive nicht gut mit ihm meint, es unterdrückt und demütigt, wenn Gefühle nicht beachtet werden. Mit zunehmender Unterdrückung entladen sie sich in Formen der Gewalt (Ideologie, Fanatismus, Amok). Eine neue Kritische Theorie muss diesen gefährlichen Anfängen auflauern, sie aufdecken und ihnen erzählerisch entgegenwirken. D.h. politische Bildungsarbeit leisten, die mehr Menschen zu erreichen vermag.

Diese Aufgabe ist nicht mit wissenschaftlichen Vorträgen und Sammelbänden getan, die die Betroffenen nicht hören, nicht lesen, nicht verstehen. Sondern durch den Dialog mit ihnen; und da der, praktisch gesehen, natürlich nicht jedes Mal direkt zu führen ist (Kluge kann ja schlecht mit Combrink, Stollmann und Schulte als platonische Propheten durch die Welt ziehen, an den Türen klopfen und die Nazis umdrehen), wird dieser Dialog durch den erzählenden wie fragmentarischen Text und Film angestoßen, die rezeptionsästhetisch betrachtet eine Appellstruktur ${ }^{33}$ besitzen, also Platz für Erfahrungen, Phantasien, Eigensinn des Rezipienten. Und zwar geschieht dies aus einer respektvollen Haltung heraus: Ihm wird etwas zugetraut - der nicht unterschätzte Mensch.

Er sagt es ja selbst, dass es ihm auf Vermittlung ankommt: Wir brauchen eine „neue Kritische Theorie“, eine „neue Enzyklopädie“, „und die muss erzählen können, die darf nicht Reden halten, die darf nicht diskursive Texte [halten], die braucht nicht den Philosophen von oben, der vom Katheder her 'was andern erklärt ““ oder eben gerade nicht erklärt. Der Mensch brauche stattdessen eine „Autonomie der Geselligkeit - und das heißt Erzählen, wie an der Theke“. ${ }^{34}$ Hieran kann man sehr gut sehen, dass das Werk Kluges nicht nur der Vermittlung von Kritischer Theorie dient (wie en passant mittels poetischer Gewandtheit), sondern auch entscheidend zum nicht immer einfachen, nicht immer widerspruchsfreien Selbstverständnis dieser beiträgt:

Kritik, d.h. Unterscheidungsvermögen, muß auf diese reale Praxis der menschlichen Intelligenztätigkeit antworten und sich deshalb in dieser Praxis selbst verankern. Sie steht

31 Vgl. Max Horkheimer, Gesammelte Schriften, hrsg. v. Alfred Schmidt u. Gunzelin Schmid Noerr, Frankfurt a. M., S. Fischer, 1987, Bd. 5, S. 64. Im Folgenden: HGS.

${ }^{32}$ Herbert Marcuse, Triebstruktur und Gesellschaft. Ein philosophischer Beitrag zu Sigmund Freud, Frankfurt a. M., Suhrkamp, 1967, S. 228.

${ }_{33} \mathrm{Vgl}$. Wolfgang Iser, Die Appellstruktur des Textes. Unbestimmtheit als Wirkungsbedingung literarischer Prosa, Konstanzer Universitätsreihe, Bd. 28, Konstanz, Universitätsverlag Konstanz, 1970.

${ }^{34}$ Andreas Ammer/Alexander Kluge/Console, Eigentum am Lebenslauf. Das Gesamte im Werk des Alexander Kluge, Produktion: Bayerischer Rundfunk, Intermedium Records, 2007, Track 9. 
dem gesellschaftlichen Prozeß nicht gegenüber. Sie kann sich deshalb nicht nur, wie im 18. Jahrhundert, schriftlich äußern oder auf eine diskutierende Öffentlichkeit vertrauen, deren Prozesse nur sehr langsam sich verbreiten, sondern sie muß selber praktische Instrumente formen, die in Form von Gegenproduktion in die Praxis einwirken. ${ }^{35}$

In gewisser Weise als Selbstreflexivum durch die freie, ungezwungene, gerade unakademische Distanz eines Ästhetischen, das über mehr Darstellungswerkzeuge verfügt als nur über Begriffskataloge. Und das heißt auch: über Komik, Trash, Unsinn, einen Alfred Edel, Helge Schneider oder Georg Schramm, wodurch bzw. durch die sich Kluge trotz aller Ernsthaftigkeit in der Sache immer eine gewisse authentische Leichtigkeit behält, die einen sowohl vor Verkrampfung im Denken wie vor Pessimismus und Resignation bewahrt. „Fröhliche Wissenschaft“, wenn man so will. Das Zwerchfell erreiche schließlich „,hohe kollektive Verständigungsstufen“ “36, besitzt also Konsensfähigkeit über alle Sprachbarrieren hinweg, wodurch das Komische dem ,bewußten Reden“"voraus ist, gar „,kritische Fähigkeiten“" ${ }^{37}$ offenbart: „Eine Sehnsucht, die sich als Albernheit kostümiert; ein energischer Wunsch, der als Understatement figuriert. Weil einer etwas was ihm wichtig ist, nicht verraten will tarnt er es ${ }^{\text {“ }}{ }^{38}$. Es zeugt von Feingeistigkeit, Kritik indirekt und mit Unterhaltungswert auszudrücken. Kluge weist aber noch auf ihre widerstandsfähige Struktur hin: Herrschaftssysteme und Autoritäten produzieren in ihrer Ernsthaftigkeit beim Versuch des totalen systemischen Selbsterhalts, in dem eigens auferlegten Zwang der Importance of Being Earnest, unfreiwillig eine Komik, die sie imstande sind, zu stürzen. ${ }^{39}$ Gefühle, Komik, Ironie, Assoziation, Erinnerung, Lachen, Weinen usw. haben eines gemeinsam: Sie verhalten sich durch ihren Charakter des Zweifelns anti-autoritär. ${ }^{40}$

Im Kampf gegen die faschistische Besetzung der Kunst, also die Ästhetisierung des Politischen, griffen Autoren wie Walter Benjamin und Bertolt Brecht zur Waffe der Politisierung der Kunst. Ästhetischer Widerstand gegen außerästhetische Wirklichkeit ist ja auch immer zugleich außerästhetischer Widerstand. Sämtliche gesellschaftlichen Verhältnisse, so die einstige Idee, sollten in der Kunst, und vordergründig in der Literatur, gespiegelt werden („Literarisierung aller Lebensverhältnisse “ $\left.{ }^{41}\right)$. Um ein solch allumfassendes Vorhaben umzusetzen, hieß es sozusagen: Schriftsteller aller Länder, vereinigt euch!

Ein Autor, der die Schriftsteller nichts lehrt, lehrt niemanden. Also ist maßgebend der Modellcharakter der Produktion, der andere Produzenten erstens zur Produktion anzuleiten, zweitens einen verbesserten Apparat ihnen zur Verfügung zu stellen vermag. Und zwar ist dieser Apparat umso besser, je mehr er Konsumenten der Produktion zuführt, kurz aus Lesern oder aus Zuschauern Mitwirkende zu machen imstande ist. ${ }^{42}$

\footnotetext{
${ }^{35} U M, M P$, S. 995 sowie die dortige Fußnote 17, die u.a. auf $U M, G E$, S. 450 ff. verweist.

36 GE, S. 383.

37 Ibid., S. 384.

38 Ibid., S. 381.

39 Ibid., S. 400.

${ }^{40}$ Und eben aufgrund ihrer „Nicht-Emanzipation“ sind sie „Gegenstand der Bearbeitung“. Vgl. GE,

${ }^{41}$ BGS II.2, S. 694.

42 Ibid., S. 696. Hervorhebung gemäß Original.
} S. 476. 
Legt man jene Schablone nun auf die Hauptperson dieser internationalen Buchausgabe, so formen sich klugesche Merkmale wie Autorenkollektiv, Autorenwerkstatt, Verlag, Film, Fernsehen der Autoren, Kollektivfilm, Kluges dctp (development company for television program mbh) der Köpfe der Zeit und seine Schreibmethode als solche. Selbst sein bedauerlicherweise unverwirklichtes Vorhaben, gemeinsam mit Arno Schmidt und anderen Groschenhefte an den Bahnhöfen der Nation zu bespielen ${ }^{43}$, gehört zu diesem Prinzip eines breiten und zugleich gehaltvollen Verlegertums, das schon Benjamin einfordert:

Die bedeutende literarische Wirksamkeit kann nur in strengem Wechsel von Tun und Schreiben zustande kommen; sie muß die unscheinbaren Formen, die ihrem Einfluß in tätigen Gemeinschaften besser entsprechen als die anspruchsvolle universale Geste des Buches in Flugblättern, Broschüren, Zeitschriftenartikeln und Plakaten abbilden. Nur diese prompte Sprache zeigt sich dem Augenblick wirklich gewachsen. ${ }^{44}$

Im Rahmen der Tagung, die diesem Band vorausging, wurde der Film Die Artisten in der Zirkuskuppel: ratlos gezeigt. Darin beschließt Leni Peickert zum Fernsehen zu gehen. Es heißt: „Sie erlernt die Technik. Sie sucht keine Karriere im redaktionellen Bereich, weil sie weiß: Hier wird man nur abhängig. Die Kenntnis der Technik versteht sie als materielle Basis ihrer gesellschaftlichen Bemühungen“". Oder: „Irgendwann einmal wächst dies zusammen: Die Liebe zur Sache, die Romane und die Fernsehtechnik“. Man ist dazu verleitet zu sagen: selbstreflexive Worte oder zumindest möglichkeits-projizierende Worte eines Alexander Kluge, der früh - der Film ist von 1968 - erkannte, dass der Weg nur über Gegenproduktion führt.

Wie man aus dem Bestand des Suhrkamp-Archivs im Deutschen Literaturarchiv Marbach entnehmen kann, hatte Kluge Ende der 70er die bereits ausgefeilte Idee, ein riesiges Kollektivprojekt der Schriftsteller und Journalisten zu begründen und konnte dies seinem Verleger Siegfried Unseld auch überzeugend vortragen: „Er entwickelte sofort einen Plan: ihm ging es um die Gründung einer Autorenwerkstatt" mit dem Ziel, eine „,Chronik der laufenden Ereignisse“" zu erstellen ${ }^{45}$. Die wichtigsten Persönlichkeiten, die die damalige Gegenwart entscheidend prägten, sollten in Interviews zu einer solchen Zeitspiegelung aufgesucht werden. Zu einer Realisierung kam es damals zwar nicht, doch hat Kluge an der Idee festgehalten und sie schließlich in den zahlreichen Formen der dctp und seiner Medienpartner verwirklicht. Ja, es steht dort Schwarz auf Weiß; es gibt ein eigenes Kapitelnetz, das da heißt: „Werkstatt der Autoren“. Und in dieser Nachrichtenwerkstatt, ist weiter zu erfahren, arbeite man „mit allen Partnern an der Aufhebung der Trennung zwischen Tatsachen, Musik, Vernunft und Emotion“. Eine Kleinigkeit zur „Spiegelung“ von Wirklichkeit sei indes nachgetragen: Im gemeinsamen Gespräch differenzierte Kluge seine Tätigkeit nicht als ein Spiegeln und auch nicht als ein Bearbeiten von Wirklichkeit (also im Sinne der Imitatio nach Horaz), sondern als eine dritte

43 Alexander Kluge, 20 Geschichten für Arno Schmidt. Ein Gruß zum 100. Geburtstag in 10 Folgen. „Folge 1: Meine Begegnung mit Arno Schmidt“, veröffentlicht am 13.01.2014. URL: http://www. logbuch-suhrkamp.de/forum/alexander-kluge-20-geschichten-fuer-arno-schmidt [Stand: 18.01.2014].

${ }^{44}$ BGS IV.1, S. 85.

45 Aus dem verlagsinternen Reisebericht von Siegfried Unseld vom 15.-19.06.1979. Vgl. Siegfried Unseld Archiv/Deutsches Literaturarchiv Marbach. 
imaginäre Wirklichkeit zwischen den Dingen - eben der „Ort der Narration“. Das involviert also auch den Optativ bzw. die Fiktion, die Fakes neben den Facts. Mimesis, nicht Imitatio. Nachahmung nach der Poetik des Aristoteles - auch das ganz im Sinne Adornos.

In diesem Zusammenhang lohnt es, zum Schluss noch einen kurzen Blick auf die Rolle der Kunst speziell für die Kritische Theorie zu werfen. Max Horkheimer etwa bestätigt im Grunde Kluges Praxis der Orchestrierung von Theorie und Poesie: „Obgleich ihr Kriterium allein in ihr selbst liegt, ist Kunst nicht weniger Erkenntnis als Wissenschaft" ${ }^{* 46}$. Ästhetische Erfahrung gilt der Kritischen Theorie als Gegenentwurf zum funktionalen Denken der instrumentellen Vernunft, da sie dazu befähigen kann, auch Bereiche außerhalb der Ratio zu erschließen. ${ }^{47}$ Die Kunst gilt dabei gewiss nicht als etwas per se Gutes oder Schlechtes, doch wird sie als ein gefährdetes, von allen Seiten beeinflussbares Territorium wahrgenommen. Nichtsdestoweniger gilt sie als ein phantastischer Ort, als virtueller Ort der Utopie, an dem zukünftige Formen gesellschaftlichen Zusammenlebens erprobt werden können. Solche IdealLeben sind ästhetisch verschränkt mit dem Real-Leben (Heterotopie). Im Kunstwerk scheint eine bessere Welt auf, und sei es nur als Negativ. Man muss sich schließlich einmal bewusst machen, dass die Kunst mehr Demokratie und mehr Humanität wagt als jede existierende Gesellschaft und jeder jemals existierte Staat, in denen Utopie und Phantasie desavouiert werden, während hingegen ihre Realität nicht weniger grausam und abgründig ist als Gewaltdarstellungen in der Kunst. ${ }^{48}$

Diese Inkonsequenz und Indifferenz, diese Wurstigkeit des modernen Menschen klagt Herbert Marcuse an: „Nur in der Kunst hat die bürgerliche Gesellschaft die Verwirklichung ihrer eigenen Ideale geduldet und sie als allgemeine Forderung ernst genommen“49. „Ästhetische Erfahrung ist [...] auch der Ort eigensinniger Wahrnehmungen, Empfindungen und Wunschproduktionen, die das Bestehende transzendieren und seine Geltung relativieren“.50 Die „Alltagsroutine“ hat Horkheimer zufolge schließlich eine „ertötende Wirkung“ auf unser „Wahrnehmungsvermögen“. ${ }^{51}$ Die epistemische Wahrnehmungskompetenz der Kunst begegnet dem Abstrakten des Realen mit der Authentizität der Phantasie und des Sinnlichen - ästhetischer vs. ideologischer Schleier. Deshalb muss Kunst als das demokratiefördernde Bildungsgut schlechthin angesehen und entsprechend gefördert werden (kulturelle und ästhetische Bildung). Das ist zumindest auf eine Weise recht nah an Schiller gebaut bzw. an dessen Briefen Über die ästhetische Erziehung des Menschen. In diesen skizziert er einen ästhetischen Staat, aber nicht als Ziel, sondern als Ideal, durch den man gehen muss, um endlich in den moralischen Staat zu gelangen, der trotz seiner Sittlichkeit „den subjektiven und spezifischen

${ }^{46}$ HGS 4, S. 420.

47 Vgl. Gerhard Schweppenhäuser, Kritische Theorie, Stuttgart, Reclam, 2010.

${ }^{48}$ So ist zum Beispiel zu bezweifeln, dass eine Vergewaltigung irgendwie ,romantischer“ abläuft als in Irréversible von Gaspar Noé.

${ }^{49}$ Herbert Marcuse, Schriften, Frankfurt a. Main, Suhrkamp, 1978-1989, Bd. 3, S. 210.

${ }^{50}$ Schweppenhäuser, Kritische Theorie, S. 22f.

51 HGS 5, S. 397. 
Charakter in den Individuen“ 52 würdigt, die Differenz. Kunst als öffentlich-kritische Plattform, als Experimentierfeld des Realen, als Korrektiv und Anklage, Pionier und Wegweiser von Wirklichkeit.

Und so gilt Adornos Satz: „Weil es in der Welt noch keinen Fortschritt gibt, gibt es einen in der Kunst; , il faut continuer." "53 Gemeint ist hier freilich der humanitäre, nicht ein technischer, wissenschaftlicher oder gar wirtschaftlicher Fortschritt. Auch ist dieser Fortschrittsgedanke nicht als linear zu begreifen, sondern er ist, da er mit ständigen Rückschlägen behaftet ist und da heterogene Gesellschaften koexistieren, unregelmäßig zirkulierend. Dieser adornosche Imperativ des Immer-Weiter künstlerischer Arbeit gegen alle Absurdität des Seins vermittelt Notwendigkeit und Hoffnung auf dessen Verwirklichung. Im Gegensatz zu einem religiösen Erlösungsszenario etwa, besteht hier der nicht metaphysische Glaube an eine humanitäre Weltgesellschaft. „Göttliches“ ist hier ein diesseitiges „Übermenschliches“, das nicht von oben, sondern von unten kommt, das der Mensch aus sich selbst heraus entwickelt. Das wird in der Kunst angedeutet und in ihr - etwa durch Kluge - lebendig aufbewahrt, und beim Revuepassieren von Geschichte durch seine Facts und Fakes rückt es faktisch und nachvollziehbar näher.

Das Bittere ist nur, dass sich das Elend so lang hinzieht und uns sehr wahrscheinlich alle, wie wir hier glücklich international zusammenkommen, überdauern wird - ,il faut continuer"...

${ }^{52}$ Friedrich Schiller, Über die ästhetische Erziehung des Menschen in einer Reihe von Briefen (1795), in ders., Schriften zur Philosophie und Kunst, München, Goldmann, 1959. Vierter Brief, S. 73.

${ }^{53}$ AGS 7, S. 310. Das innere Zitat stammt von Samuel Beckett. Eine Deutung, weshalb Adorno gerade ihn hier verwendet, könnte sein, dass es ein Wink auf das „Absurde“ ist, in dem Fall die Absurdität, die Unvernünftigkeit der Einrichtung von Welt. Dies würden zumindest die folgenden Zeilen aus der Ästhetischen Theorie unterstützen: „Vorrang des Objekts und ästhetischer Realismus sind heute fast kontradiktorisch einander entgegengesetzt, und zwar nach realistischem Maß: Beckett ist realistischer als die sozialistischen Realisten, welche durch ihr Prinzip die Wirklichkeit verfälschen. Nähmen sie diese streng genug, so näherten sie sich dem, was Lukács verdammt, der während der Tage seiner Haft in Rumänien geäußert haben soll, nun wisse er, daß Kafka ein realistischer Schriftsteller sei."Vgl. AGS 7, S. 477 . 
